\title{
Efficacy of serum miR-106b and miR-222 as non-invasive diagnostic markers for hepatocellular carcinoma in $\mathrm{HCV}$-infected Egyptian patients
}

\author{
Azza A. Atef ${ }^{1}$, Abdel-Hady A. Abdel-Wahab ${ }^{2}$, Emad K. Ahmed ${ }^{1 *}$ and Rehab Abdel-Hameed ${ }^{1}$

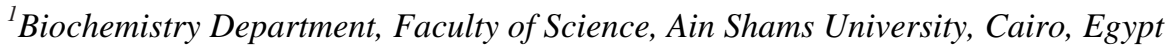 \\ ${ }^{2}$ Cancer Biology Department, National Cancer Institute, Cairo University, Giza, Egypt
}

ARTICLE INFO

Article history:

Received 31 May 2016

Accepted 27 June 2016

Keywords:

Hepatocellular carcinoma;

$\mathrm{HCV}$;

MicroRNA;

Diagnostic;

Biomarkers.

\begin{abstract}
A B S T R A C T
MicroRNAs (miRNAs) have been shown to anticipate great cancer diagnostic potential. Recently, circulating miRNAs have been reported as promising biomarkers for various pathologic conditions including hepatocellular carcinoma (HCC). HCC development was found to be associated with the major risk factors Hepatitis B virus (HBV) and Hepatitis C virus (HCV). The current study was initiated to evaluate the diagnostic potential of a panel of five circulating, HCCrelated miRNAs as biomarkers of $\mathrm{HCC}$ among $\mathrm{HCV}$-infected Egyptian patients. Levels of serum miRNAs were measured using qRT-PCR in 25 HCC-post HCV infected, $52 \mathrm{HCV}$-positive and 50 control individuals. In addition, the expression levels of the chosen miRNAs were also assessed in liver tumor tissue samples of patients in the HCC group. MiR-21, miR-30c and miR-222 were significantly upregulated whereas miR-106b was downregulated in sera of HCC cases compared to controls. Also, miR-21, miR-93, and miR-222 were overexpressed whereas miR-30c was down-expressed in $\mathrm{HCV}$-infected patients, compared to controls. Statistical analyses have revealed a differential expression in the level of serum miR-21, miR-30c, miR-106b, and miR-222 between the HCC and HCV patient groups. Concordance in expression state between serum and tissue samples of HCC was only observed for miR-106b and miR-222. The sensitivity and specificity of miR-106b for detecting $\mathrm{HCC}$ among $\mathrm{HCV}$-positive individuals was found to be $46.2 \%$ and $84 \%$, respectively. Whereas, the sensitivity and specificity of miR-222 were $72 \%$ and $59.6 \%$, respectively. On the other hand, a significant correlation was detected between the relative expression level of miR222 and liver cirrhosis $(\mathrm{P}=0.048)$. No correlation was detected between the relative expression level of miR-106b and clinicopathological parameters in HCC group. In addition, a significant correlation $(\mathrm{P}=0.001)$ was detected between the expression levels of the two miRNAs. In conclusion, the present study shows that miR-106b and miR-222 are differentially expressed in HCC-post HCV and HCVpositive patient samples and that both of them have some diagnostic potential for detecting primary HCC in chronically HCV-infected patients. Further studies are needed to evaluate the diagnostic power of circulating miR-106b and miR-222 for hepatocellular carcinoma among high-risk HCV Egyptian patients.
\end{abstract}

\section{Introduction}

Hepatocellular carcinoma (HCC) is the most common type of primary liver cancer, accounting for $\sim 85 \%$ of all cases ${ }^{[1]}$. Overall, it is the fifth most common cancer worldwide, representing about $5.7 \%$ of all new human cancer cases with an increasing incidence expected in

* Corresponding author.

E-mail address: emad.ahmed@sci.asu.edu.eg the next decade ${ }^{[2]}$. Approximately $80-90 \%$ of patients with HCC have the underlying viral etiologies. From a global perspective, the most important risk factors for HCC are Hepatitis B virus (HBV) and Hepatitis C virus $(\mathrm{HCV})^{[3]}$. It has been reported that Egypt has the highest prevalence of HCV worldwide and most of HCC cases ( 94\%) are associated with chronic HCV infection ${ }^{[4]}$.

The major potentially curative form of therapy is still 
surgical resection, however only $10 \%$ of patients were at operable stages upon disease discovery. This is mainly due to the absence of reliable tools for early diagnosis and thus most patients were at a late stage either for surgical solutions or for effective treatment ${ }^{[5]}$. The widely used serological tumor markers for $\mathrm{HCC}, \alpha-$ fetoprotein (AFP) and des- $\gamma$-carboxy prothrombin (DCP) have unsatisfactory specificity and sensitivity. AFP specificity and sensitivity are $75 \%$ and $68 \%$, respectively, whereas elevated DCP activity is only present in 44-47\% of HCCs less than $3 \mathrm{~cm}$ in size ${ }^{[6]}$.

MicroRNAs (miRNAs) are endogenous, small, singlestranded, noncoding RNAs that consist of 20 to 25 bases. These control various biologic functions such as cellular proliferation, differentiation, and apoptosis. Approximately half of human miRNAs are located in fragile regions of chromosomes, which are associated with the development of human cancers. MiRNAs have been referred to as so-called oncomirs for their functions as tumor suppressors and oncogenes ${ }^{[7]}$. Aberrant expression of miRNA has been linked to a variety of cancers, including $\mathrm{HCC}^{[8]}$.

Investigation of cancer-specific miRNAs in the circulation is an emerging and exciting field of study. One of the first studies measuring miRNA levels in serum was reported by Lawrie et al. ${ }^{[9]}$ in 2008. Subsequently, circulating miRNAs have been postulated as novel biomarkers for cancer, and other disease processes ${ }^{[10]}$. Recently, several studies have pointed out the potential of using miRNAs as non-invasive biological markers to screen high-risk patients in order to enhance HCC detection, earlier intervention and increase the probability of successful treatment ${ }^{[8]}$. In the present study, we evaluated the possibility of using a panel of 5 circulating, HCC-related miRNAs as putative non-invasive biomarkers for screening high-risk $\mathrm{HCV}$ infected Egyptian patients for the early detection of HCC using qRT-PCR.

\section{Subjects and Methods}

Twenty-five primary $\mathrm{HCC}$ patients with past $\mathrm{HCV}$ infection, 52 chronic HCV-infected patients, and 50 ageand sex- matched healthy subjects (pooled in 10) were participated in this study (Table 1). The study protocol was performed according to the guideline for the use of human subject's materials and approved by the Cairo university hospital research ethics committee (REC), school of medicine, Cairo University. Additionally, discussion with the subjects regarding the benefits and hazards of the study was done and informed written consent was obtained from participants for the use of their blood and tissue samples in this study. HCC patients were selected from outpatient Clinic of Radio Diagnosis Department, National Cancer Institute (NCI), Cairo University (Cairo, Egypt). HCV patients were recruited from Kasr El-Aini, Tropical Medicine Department, Faculty of Medicine, Cairo University.

HCC patient's selection criteria were high serum level of $\alpha$-Fetoprotein (AFP), showing criteria of HCC in tri- phase CT scan. Pathology examination confirms HCC disease, with no evidence of local invasion or distant metastasis and positive for HCV. Patients with cancer rather than HCC, patients with secondary $\mathrm{HCC}$ and patients with inflammation only or benign liver disease were excluded.

Three milliliter peripheral blood was collected from each patient and healthy control donors. Serum was separated for miRNAs purification. All the samples were classified into 3 groups based on a questionnaire filled by the clinician responsible for each patient.

Tumor tissues were obtained from patients in the HCC group while normal liver biopsies were obtained from 6 healthy volunteers in transplantation surgery under the approval of ethics. Collected tissues were immediately cut into two parts; one was preserved in $10 \%$ neutral formalin buffer for histopathological examination. The second part was collected in RNA-later solution (Qiagen, Germany) and was then stored at $-80^{\circ} \mathrm{C}$ for further extraction of RNA. Tissue samples were examined by the pathologist in the surgical pathology department, NCI. The existence of HCC, grade and stage determination were done for all patients.

\section{Extraction of total RNA from blood and tissue samples}

Total RNA was isolated from HCC, HCV and control serum samples using miRNeasy Serum/Plasma Kit (Qiagen, Germany) [cat. \# 217184]. Total RNA was isolated from HCC tissue and normal tissue samples using miRNeasy mini Kit (Qiagen, Germany) [cat. \#. 217004]. Blood samples tubes were centrifuged at 1200 $\mathrm{xg}$ for 10 minutes to isolate serum from the clot. Serum samples were centrifuged for $10 \mathrm{~min}$ at $9600 \mathrm{x}$ g to remove additional cellular debris and minimize contamination of cell-free nucleic acids by genomic DNA and RNA derived from damaged blood cells. For RNA isolation, $200 \mu$ l of serum was homogenized in 1 $\mathrm{ml}$ Qiazol lysis reagent. Then $200 \mu \mathrm{l}$ chloroform were added to the tube containing the lysate and centrifuged for $15 \mathrm{~min}$ at $12,000 \mathrm{x} \mathrm{g}$ at $4^{\circ} \mathrm{C}$. The upper aqueous phase was transferred to a new collection tube. $900 \mu$ l of $100 \%$ ethanol were added and mixed thoroughly by pipetting up and down several times. $700 \mu \mathrm{l}$ of the sample were pipetted up, including any precipitate that may have formed, into an RNeasy MinElute spin column in a $2 \mathrm{ml}$ collection tube and centrifuged at $8000 \mathrm{x}$ g for $15 \mathrm{~s}$ at room temperature $\left(15-25^{\circ} \mathrm{C}\right)$. The flow-through was discarded. The previous step was repeated using the remainder of the sample. The flow through was discarded. $700 \mu \mathrm{l}$ buffer RWT were added to the RNeasy MinElute spin column and tube centrifuged for $15 \mathrm{~s}$ at $8000 \mathrm{x} \mathrm{g}$ to wash the column. The flow-through was discarded. $500 \mu \mathrm{l}$ buffer RPE were pipetted onto the RNeasy MinElute spin column and the tube was centrifuged for $15 \mathrm{~s}$ at $8000 \mathrm{x} \mathrm{g}$ to wash the column. The flow-through was discarded. The RNeasy MinElute spin column was transferred to a new $1.5 \mathrm{ml}$ collection tube and $35 \mu \mathrm{l}$ RNase-free water was pipetted directly to the center of the spin column membrane and the column 
centrifuged for $1 \mathrm{~min}$ at full speed to elute the RNA. For tissue samples, the biopsy samples were stored at $-80^{\circ} \mathrm{C}$, extraction of RNA was done by adding $25 \mathrm{mg}$ of liver tissue to $700 \mu \mathrm{l}$ Qiazol lysis reagent for homogenization. A volume of $140 \mu \mathrm{l}$ of chloroform was added to the homogenate, the sample is then processed as serum samples protocol mentioned above. The concentration and quality of RNA was measured by NanoDrop 2000 spectrophotometer, Thermo Scientific, USA. RNA is then stored at $-80^{\circ} \mathrm{C}$ for further reverse transcription.

II. Reverse transcription and Quantitative (realtime) Polymerase Chain Reaction (qRT-PCR)

The extracted total RNA from serum of HCC, HCV and healthy control samples as well as that extracted from tissue samples were then reverse transcribed using miScript II RT Kit (Qiagen, Germany) [cat. \# 218161] and incubated for $60 \mathrm{~min}$ at $37^{\circ} \mathrm{C}$ followed by incubation for $5 \mathrm{~min}$ at $95^{\circ} \mathrm{C}$ to inactivate miScript Reverse Transcriptase Mix and placed on ice. The concentration of the cDNA samples is then measured by the NanoDrop using $1 \mu$ of undiluted cDNA sample. The cDNA samples are then diluted to a final concentration of 100 $\mathrm{ng} / \mu \mathrm{l}$. For qRT-PCR using miScript SYBR® Green PCR Kit (Qiagen, Germany) [cat. no. 218075] and miScript Primer Assays (Qiagen,Germany), $1 \mu \mathrm{L}$ diluted RT products were mixed with $5 \mu \mathrm{L}$ of $2 \mathrm{x}$ QuantiTect SYBR Green PCR Master Mix, $1 \mu \mathrm{L}$ 10x miScript universal primer, $1 \mu \mathrm{L}$ of $10 \mathrm{x}$ miScript primer assay and $2 \mu \mathrm{L}$ RNase-free water in a final volume of $10 \mu \mathrm{L}$ according to manufacturer instructions. All reactions were run on the Applied Biosystem using the following conditions: $95^{\circ} \mathrm{C}$ for $15 \mathrm{~min}$, followed by 40 cycles at $94^{\circ} \mathrm{C}$ for $15 \mathrm{~s}$, and $55^{\circ} \mathrm{C}$ for 30 seconds, $70^{\circ} \mathrm{C}$ for 30 seconds. Relative expression of miRNA was calculated using the comparative cycle threshold $\left(\mathrm{C}_{\mathrm{T}}\right)$ Livak's $2^{-\Delta \Delta C t}$ equation ${ }^{[11]}$ with miR-16 as the endogenous control to normalize the data. The $\mathrm{Ct}$ or threshold cycle value is the cycle number at which the fluorescence generated within a reaction crosses the fluorescence threshold in $q R T$ PCR. $\Delta \mathrm{C}_{\mathrm{T}}$ was calculated by subtracting the $\mathrm{C}_{\mathrm{T}}$ values of miR-16 from the $\mathrm{C}_{\mathrm{T}}$ values of the chosen miRNA. $\Delta \Delta \mathrm{C}_{\mathrm{T}}$ was then calculated by subtracting mean $\Delta \mathrm{C}_{\mathrm{T}}$ of the control samples from $\Delta \mathrm{C}_{\mathrm{T}}$ of tested samples. Fold change of miRNA was calculated by the equation $2^{-\Delta \Delta C T}$. A 2-fold increased $(\geq 2)$ or decreased $(\leq 0.5)$ value was chosen and considered miRNA over-expression or downregulation, respectively, in $\mathrm{HCC}$ - postHCV and $\mathrm{HCV}$ samples. Melting curve analysis of the PCR product(s) was performed to verify their specificity and identity (melting curve analysis is an analysis step built into the software of real-time cyclers).

\section{Statistical analysis}

Data were summarized using mean \pm standard error mean for quantitative variables and frequencies (number of cases) and relative frequencies (percentages) for categorical variables. Mann-Whitney test was applied when comparing fold change between two groups. Receiver Operating Characteristic (ROC) curves were constructed and the area under the curve (AUC) was calculated for the detection of the sensitivity and specificity of miRNAs for predicting HCC cases among $\mathrm{HCV}$-infected patients. Correlation between the miRNAs

Table 1: Clinicopathological parameters of the subjects involved in this study.

\begin{tabular}{|c|c|c|c|}
\hline & HCV group & HCC group & Control Group \\
\hline No. of patients & 52 & 25 & $\begin{array}{c}50 \\
\text { (pooled into 10) }\end{array}$ \\
\hline $\begin{array}{c}\text { Age (year) } \\
\text { Mean } \pm \text { S.E.M.) }\end{array}$ & $41 \pm 1.8$ & $60.72 \pm 1.5$ & $34.3 \pm 1.4$ \\
\hline $\begin{array}{c}\text { Sex } \\
\text { Male } \\
\text { Female }\end{array}$ & 40 & 20 & 38 \\
\hline HCV RNA* & + Ve & + Ve & - Ve \\
\hline AFP (ng/ml) $\geq 200$ & $0(0 \%)$ & $11(44 \%)$ & - \\
\hline HBV, N (\%) & - & $8(32 \%)$ & - \\
\hline Bilhariziasis, N (\%) & - & $19(76 \%)$ & - \\
\hline Necrosis, N (\%) & - & $13(52 \%)$ & - \\
\hline Fibrosis, N (\%) & - & $23(92 \%)$ & - \\
\hline Cirrhosis, N (\%) & - & $11(44 \%)$ & - \\
\hline AST (U/L) $\geq 40$ & - & $18(72 \%)$ & - \\
\hline ALT (U/L) $\geq 40$ & - & $17(68 \%)$ & - \\
\hline Grade, N (\%) & & $3(12 \%)$ & - \\
I & - & $19(76 \%)$ & \\
\hline III & & $3(12 \%)$ & \\
\hline
\end{tabular}


expression levels and clinico-pathological factors of HCC group was done using Spearman correlation. All statistical tests were two-sided, and a probability level of $\mathrm{p}<0.05$ was considered to be statistically significant. Data analysis was done using Statistical Package for Social Science (SPSS software package, version 20.0) program (SPSS Inc., Chicago, IL, USA).

\section{Results}

Patient Characteristics: A total of 127 participants including $25 \mathrm{HCV}$-positive $\mathrm{HCC}$ patients, 52 patients with chronic $\mathrm{HCV}$ infection and without $\mathrm{HCC}$, and 50 normal subjects were recruited into this study (Table 1). With regard to clinico-pathologic characteristics of HCC patients, the majority of patients $(92 \%)$ were fibrotic, $44 \%$ were with cirrhosis, and $52 \%$ were with hepatic necrosis. About $76 \%$ of the studied patients were with Bilharziasis while $32 \%$ were with $\mathrm{HCV} / \mathrm{HBV}$ coinfection. In addition, nearly all the HCC patients were at grade II $(92 \%)$ with just 3 out of 25 patients at grade I and 3 patients at grade III.

Expression levels of miRNAs in HCC serum samples: Of the 5 miRNAs analyzed in HCC serum samples, one miRNA was significantly down-regulated (fold change $<0.5$ ) namely miR-106b with mean fold change of 0.16 . Three miRNAs showed up-regulation in their expression (fold change > 2) namely miR-21, miR-30c and miR222, their recorded mean fold changes were 18.08, 9.28 and 18.08 respectively. Finally, one miRNA didn't show any significant changes (mean fold change is 1.88) namely miR-93 (Table 2 \& Figure 1).

Expression levels of miRNAs in HCC tissue samples: Gene expression of miRNAs was analyzed using RNA isolated from tissue biopsies from the same HCC patients. Six normal tissue biopsies were used as control. In the tissue samples, two miRNAs (miR-30c and miR106b) showed down-regulation in their expression and their fold changes were 0.12 and 0.28 respectively. Two
miRNAs (miR-93 and miR-222) showed up-regulation and their mean fold changes recorded were 6.96 and 6.88 respectively. Whereas, miR-21 didn't show any significant alteration (fold change was 0.84) (Table 2 \& Figure 1).

Expression levels of miRNAs in HCV serum samples: Of the 5 miRNAs analyzed in HCV serum samples, one miRNA was significantly down-regulated (fold change < 0.5 ) namely miR-30c with mean fold change of 0.23 . Three miRNAs showed up-regulation in their expression (fold change > 2) namely miR-21, miR-93 and miR-222, their recorded mean fold changes were 2.44, 4.77 and 8.21 respectively. Finally, one miRNA didn't show any significant changes (mean fold change is 0.87 ) namely miR-106b (Table 2 \& Figure 1).

Differential Expression of miRNAs between HCC and HCV serum samples: To evaluate the difference in the miRNAs expression level between $\mathrm{HCV}$ and HCC groups, Non-parametric Mann-Whitney U test was conducted and results are shown in Table (2). Test results revealed that, of the 5 miRNAs studied, 4 miRNAs were found to be significantly deregulated $(\mathrm{p}<0.05)$ in $\mathrm{HCC}$ group namely miR-21, miR-30c, miR$106 \mathrm{~b}$ and miR-222, compared to HCV group. Of these 4 deregulated miRNAs, 3 miRNAs were significantly elevated namely miR-21, miR-30c and miR-222 while miR-106b was significantly down-regulated. Meanwhile no significant change was observed in the expression level of miR-93 between the 2 groups (Table 2).

Correlation between serum miR-106b \& miR-222 and clinical data of HCC patients: No correlation was detected between the relative expression level of miR$106 \mathrm{~b}$ and clinico-pathological parameters. Only a significant correlation was observed between the expression level of miR-222 and liver cirrhosis $(\mathrm{P}=$ 0.048). In addition, a highly significant correlation $(\mathrm{P}<0.001)$ was detected between the expression levels of the 2 miRNAs (Table 3).

Table 2: The relative expression levels of the studied miRNAs in HCV (serum) and HCC (serum \& tissue) samples.

\begin{tabular}{|c|c|c|c|c|}
\hline Group & $\begin{array}{c}\text { HCV } \\
\text { (serum) }\end{array}$ & $\begin{array}{c}\text { HCC } \\
\text { (serum) }\end{array}$ & $\begin{array}{c}\text { Mann-Whitney } \\
\text { (Significance) }\end{array}$ & $\begin{array}{c}\text { HCC } \\
\text { (tissue) }\end{array}$ \\
\hline miR-21 & $\begin{array}{c}2.44 \pm 0.75 \\
\text { (up) }\end{array}$ & $\begin{array}{c}18.08 \pm 5.10 \\
\text { (up) }\end{array}$ & $<0.001^{* *}$ & $\begin{array}{c}0.84 \pm 0.26 \\
\text { (N.S.) }\end{array}$ \\
\hline miR-30c & $\begin{array}{c}0.23 \pm 0.13 \\
\text { (down) }\end{array}$ & $\begin{array}{c}9.28 \pm 3.09 \\
\text { (up) }\end{array}$ & $<0.001^{* *}$ & $\begin{array}{c}0.12 \pm 0.07 \\
\text { (down) }\end{array}$ \\
\hline miR-93 & $\begin{array}{c}4.77 \pm 1.47 \\
\text { (up) }\end{array}$ & $\begin{array}{c}1.88 \pm 0.54 \\
\text { (N.S) }\end{array}$ & N.S. & $\begin{array}{c}6.96 \pm 3.54 \\
\text { (up) }\end{array}$ \\
\hline miR-222 & $\begin{array}{c}0.87 \pm 0.17 \\
\text { (N.S.) }\end{array}$ & $\begin{array}{c}0.16 \pm 0.08 \\
\text { (down) }\end{array}$ & $<0.01^{*}$ & $\begin{array}{c}\text { (up) } \\
\text { (down) }\end{array}$ \\
\hline
\end{tabular}

Results are expressed as mean \pm S.E.M.

* : Significant at significance level $\mathrm{P}<0.01$

**: Significant at significance level $\mathrm{P}<0.001$

N.S.: Non-Significant. 


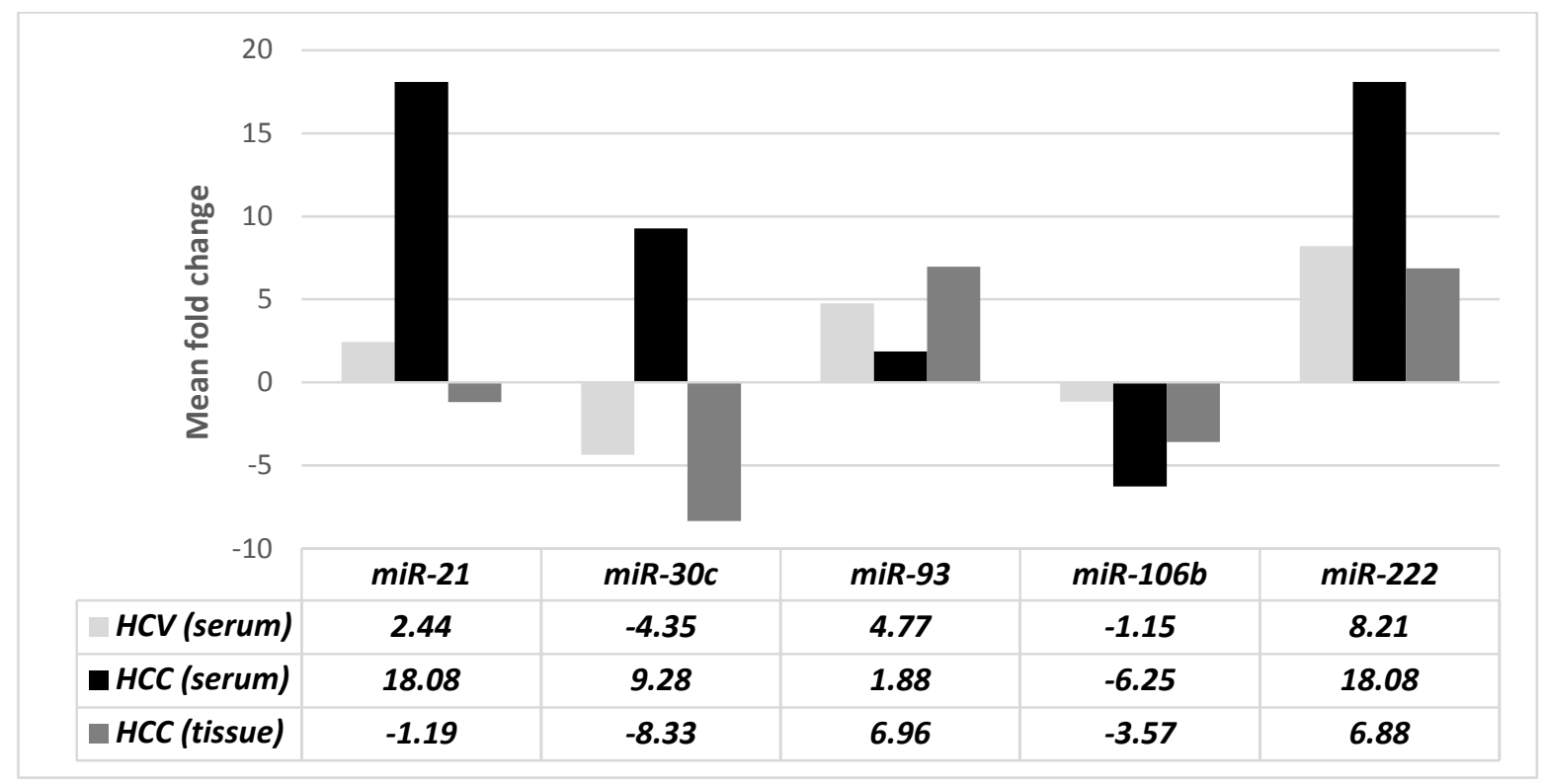

Figure (1): The mean fold changes of miRNAs in HCV (serum) and HCC (serum and tissue) samples. Expression levels of miR-21, miR-30c, miR-93, miR-106b, and miR-222 in HCC (serum/tissue) and HCV serum samples. MiR-16 was used as normalization control.

Table 3: Correlation between the relative expression levels of miR-106b and miR-222 with clinical data in HCC group.

\begin{tabular}{|c|c|c|c|c|}
\hline \multirow{2}{*}{ Parameter } & \multicolumn{2}{|c|}{ miR-106b } & \multicolumn{2}{c|}{ miR-222 } \\
\cline { 2 - 5 } & $\begin{array}{c}\text { Correlation } \\
\text { Coefficient } \\
\text { (r) }\end{array}$ & p-value & $\begin{array}{c}\text { Correlation } \\
\text { Coefficient } \\
\text { (r) }\end{array}$ & p-value \\
\hline Gender & -0.218 & 0.295 & 0.258 & 0.213 \\
\hline Age & -0.129 & 0.539 & -0.159 & 0.448 \\
\hline HBV & 0.168 & 0.421 & 0.114 & 0.589 \\
\hline Bilharziasis & -0.010 & 0.961 & -0.105 & 0.619 \\
\hline AST & 0.083 & 0.692 & -0.002 & 0.994 \\
\hline ALT & 0.076 & 0.719 & -0.128 & 0.543 \\
\hline AFP & 0.273 & 0.187 & 0.177 & 0.398 \\
\hline Grade & 0.223 & 0.285 & 0.296 & 0.151 \\
\hline Necrosis & -0.236 & 0.256 & -0.201 & 0.335 \\
\hline Fibrosis & -0.273 & 0.186 & -0.247 & 0.234 \\
\hline Cirrhosis & -0.387 & 0.056 & $-0.399^{*}$ & 0.048 \\
\hline MiR-106b & - & - & $0.624^{* *}$ & 0.001 \\
\hline MiR-222 & $0.624^{* *}$ & 0.001 & - & - \\
\hline
\end{tabular}

*Statistically significant correlation.

** Highly statistically significant correlation.

Diagnostic potential of circulating miR-106b, miR222 and AFP in HCC patient group: To characterize the diagnostic value of miR-106b and miR-222, ROC curves were constructed and the AUC was calculated to evaluate the sensitivity and specificity for predicting $\mathrm{HCC}$ cases among HCV-infected patients based on the expression of each individual miRNA. ROC curves showed that a cut-off value of 0.5 yielded the best sensitivity (46.2\%) and specificity $(84.0 \%)$ for miR-106b relative gene expression while a cut-off value of 3.5 yielded the best sensitivity $(72.0 \%)$ and specificity $(59.6 \%)$ for $\mathrm{miR}-222$ relative gene expression in differentiating patients with HCC from those with HCV. On the other hand, The ROC curve showed that a cut-off value of 10.5 yielded the best sensitivity (96.0\%) and specificity $(86.5 \%)$ for AFP level in differentiating patients with HCC from those with HCV (Figure 2). 


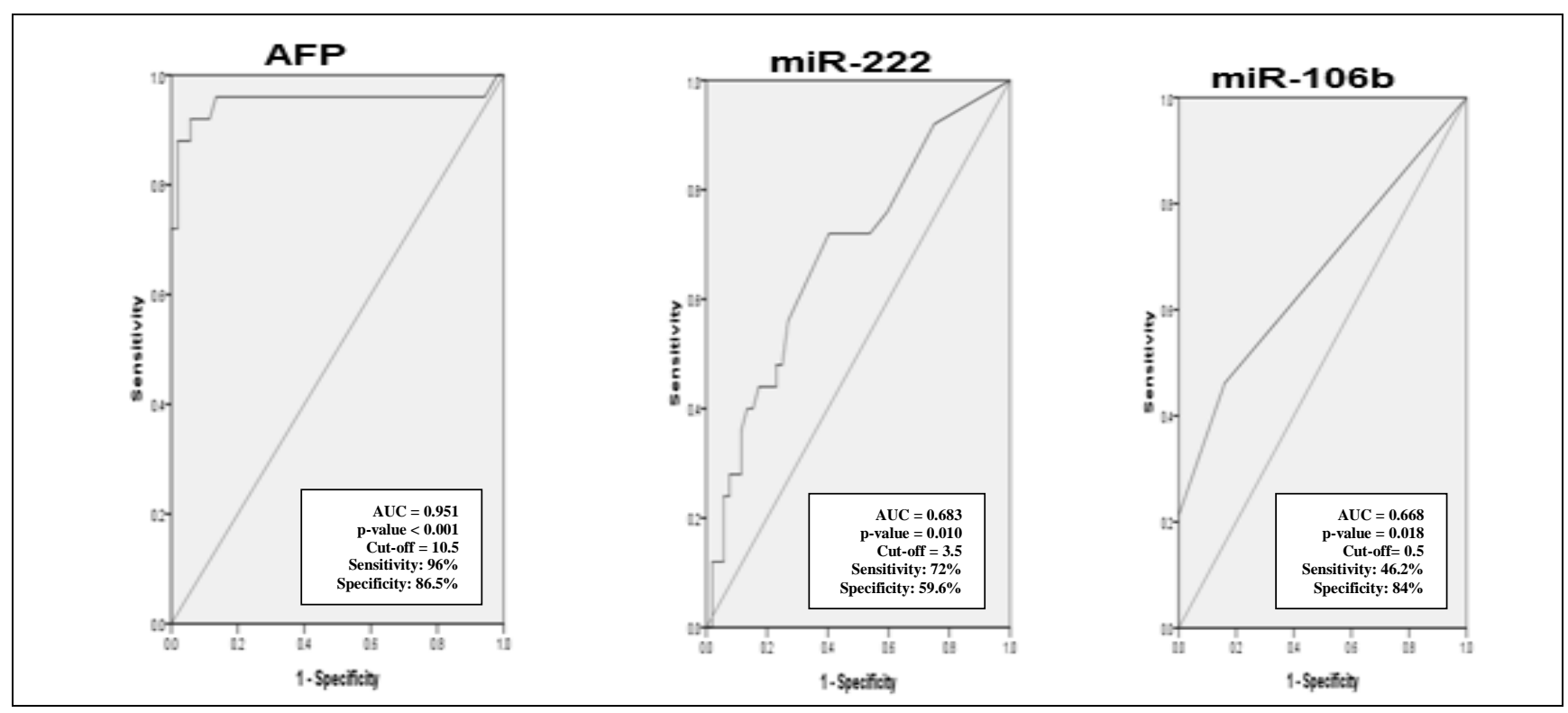

Figure (2): ROC curves of miR-106b, miR-222 relative expression and AFP level between HCV and HCC groups. Receiver operating characteristics (ROC) curves for serum miR-106, miR-222 and AFP for discriminating HCC patients from HCV patients without HCC.

\section{Discussion}

There is now mounting evidence indicating that specific miRNAs may act as oncogenes or tumor suppressors by directly or indirectly controlling the expression of key proteins involved in hepatocarcinogenesis ${ }^{[12,13]}$. From a diagnostic point of view, high stability of miRNAs in circulation makes them perfect biomarkers. However, lack of studies on preneoplastic and early neoplastic lesions does not allow understanding whether miRNAs are directly involved in hepatocarcinogenesis or deregulated due to disease activity ${ }^{[14]}$. The aim of the current study was to investigate the potential of using deregulation of miRNA expression in HCC-postHCV infected patients for the early diagnosis of HCC in the high-risk HCVinfected Egyptian patients.

In the present study, the serum levels of 5 miRNAs namely miR-21, miR-30c, miR-93, miR-106b and miR222 were analyzed in patients with HCC, HCV and controls and their diagnostic performance in the context of AFP, the conventional diagnostic marker of HCC, was evaluated. Furthermore, the levels of theses miRNAs were analyzed in HCC tissue biopsies in order to find the relation between the expression pattern of these miRNAs in serum and tissue in the same patients. In HCC patients, serum levels of miR-21, miR-30c and miR-222 were elevated while miR-106b was decreased compared to healthy controls. In patients with HCV infection, miR-21, miR-93 and miR-222 serum levels were elevated while serum level of miR-30c was decreased compared to healthy controls. To evaluate the difference in the miRNAs expression level between HCV group and HCC group, Non-parametric MannWhitney U test was conducted. Test results revealed that, of the 5 miRNAs studied, 4 miRNAs were found to be significantly deregulated $(\mathrm{p}<0.05)$ in $\mathrm{HCC}$ group namely miR-21, miR-30c, miR-106b and miR-222, compared to HCV group. Of these 4 significant miRNAs; 3 miRNAs were significantly elevated namely miR-21, miR-30c and miR-222 while the remaining one (miR-106b) was significantly down-regulated in HCC group compared to $\mathrm{HCV}$ patient group. In order to investigate if any one of these 5 significantly deregulated miRNAs has a potential to be used as a biomarker for HCC detection among HCV-infected patients, expression state of these miRNAs in HCC serum samples was compared with their relatives in HCC paired tissue samples. From comparison, miR-21 was excluded since it was up-regulated in HCC tissue samples while being not significantly changed in their paired serum samples. In a similar way, miR-30c was also excluded since its expression in HCC serum samples is up-regulated while being down-regulated in their paired tissue samples. Thus, it was decided to study the potential of the remaining 2 miRNAs; miR-106b and miR-222 in the early detection of HCC among high-risk HCV infected Egyptian patients.

To study the diagnostic potential of miR-106b and miR222 for discriminating HCC patients from HCV infected patients, ROC curve analysis was used. Results of the ROC curve analysis of miR-106b revealed an AUC of 0.668 with $46.2 \%$ sensitivity and $84.0 \%$ specificity. MiR-222 revealed an AUC of 0.683 with $72.0 \%$ sensitivity and $59.6 \%$ specificity for discriminating HCC from $\mathrm{HCV}$-infected patients. To further explore the diagnostic potential of miRNA-106b and miR-222, The AUC values on the ROC curve for serum miRNA-106b and miR-222 were compared with that of AFP, the classical HCC Classifier.

Interestingly, the AUC of AFP (0.951) in the present 
study was much higher than the range previously reported $(0.712-0.830)^{[15-17]}$. Luo et al ${ }^{[18]}$ reasoned the high AUC of AFP (0.935) observed in their study to that most of their patients had late-stage $\mathrm{HCC}$, and therefore had a significantly higher AFP level. Unfortunately, no data was available about the stage status of HCC patients participated in the current study. Although miR-106b and miR-222 have lower AUC than AFP, the diagnostic performance of miRNA-106b and miR-222 in the present study was comparable with that previously reported for AFP (AUC 0.712-0.830), AFP immunocomplex (AUC 0.691), squamous cell carcinoma antigen (AUC 0.703), and squamous cell carcinoma immunocomplex (AUC 0.694) in patients with $\mathrm{HCC}^{[19,20]}$. In the present study and by combining either the miRNA-106b or the miR-222 levels with the AFP level, the sensitivity was notably increased (100\%) compared with AFP alone (data not shown), suggesting that miRNA-106b and miR-222 may have some diagnostic potential. However, it can be noticed that the diagnostic power of AFP was significantly superior to that of either miRNA-106b or miR-222. Also, the combined classifiers did not result in significantly better performance than AFP alone. These findings establish that both serum miRNA-106b and miR-222 have some diagnostic potential, both individually and in combination with AFP.

MiR-106b, as a member of the miR-106b-25 cluster, is known to promote cancer cell proliferation and survival in gastric cancer and hepatocellular carcinoma ${ }^{[21-23]}$. It was also found to be frequently dysregulated in many human cancers, including colorectal cancer ${ }^{[24]}$. MiR222 is a well-studied oncogenic microRNA that is frequently upregulated in several types of human tumors, such as esophageal adenocarcinoma, gastric adenocarcinoma, colorectal adenocarcinoma, hepatocellular carcinoma, and pancreatic ductal adenocarcinoma. In these cancers, silencing of miR-222 could represent a novel anti-tumor approach to inhibit tumor growth and metastasis. On the other hand, miR222 also plays onco-suppressive roles in cholangiocarcinoma and gastrointestinal stromal tumors [25].

The present results regarding the expression of miR222 go hand in hand with several previous studies which reported up-regulation of miR-222 in primary HCC tumors ${ }^{[26-28]}$. In addition, it has been found that circulating miR-222 was up-regulated in the serum/plasma of HCC patients carrying the hepatitis B or $\mathrm{C}$ virus ${ }^{[28,29]}$.

In contrast to current findings regarding the expression of miR-106b, previous studies have shown that miR$106 \mathrm{~b}$ expression is up-regulated in HCC tissue specimens and detection of its expression could distinguish HCC tissues from adjacent noncancerous tissues [23,30,31]. However, to date, few studies have reported serum levels of miR-106b in HCC in the literature. Furthermore, no study has compared serum levels of miR-106b among normal, HCV-infected and
HCC patients.

Connolly et al. ${ }^{[32]}$ have shown that the expression level of miR-106b in human HCC tissue with HBV infection was increased compared with the non-tumor liver tissue of the controls. In the present study, the expression of miR-106b is down-regulated in HCC samples (serum and tissue) while it does not exhibit significant change in $\mathrm{HCV}$ serum samples. Compared with previous studies, the current results are mostly not-consistent with those obtained for tissue microRNAs in patients with HBV-related HCC suggesting that the upregulation observed in these studies may be due to HBV infection.

In conclusion, circulating miR-21, miR-30c, miR-106b and miR-222 are differentially expressed in HCCpostHCV and HCV-infected patients. Concordance was observed in miR-106b and miR-222 expression state between serum and tissue samples in HCC patients. The serum level of miR-106b has moderate sensitivity and high specificity while miR-222 has moderate specificity and sensitivity in HCC detection among HCV-infected Egyptian patients. In addition, by combining either the miRNA-106b or the miR-222 levels with the AFP level, the sensitivity was notably increased compared with AFP alone, suggesting that both serum miRNA-106b and miR-222 have some diagnostic potential, both individually and in combination with AFP. Serum miR$106 \mathrm{~b} \&$ miR-222 levels do not correlate with many of the clinicopathological characteristics of $\mathrm{HCC}$ patients. The use of miR-106b or miR-222 as diagnostic tools for $\mathrm{HCC}$ among HCV-positive patients is not as powerful as AFP. Further analysis is needed to evaluate the diagnostic power of circulating miR-106b and miR-222 for hepatocellular carcinoma among $\mathrm{HCV}$-infected Egyptian patients. Future studies will focus on developing a clinically useful early diagnostic test for HCC using these biomarkers and will validate the current data in a larger sample size study.

\section{Acknowledgement}

The authors want to thank Dr. Ekram Hamed, Professor of Interventional Radiology, RadioDiagnostic department, National Cancer Institute for providing the HCC tissue samples used in this study.

\section{References}

1) Columbo, M. In: Sorrell, M. F., Schiff, E. R. and Maddrey, W. C. (2003). Diseases of the liver. Philadelphia: Lippincott Williams \& Wilkins; pp.1378-1391.

2) El-garem, H., Ammer, A., Shehab, H., Shaker, O., Anwer, M., El-akel, W. and Omar, H. (2014). Circulating microRNA, miR-122 and miR-221 signature in Egyptian patients with chronic hepatitis C related hepatocellular carcinoma. World J. Hepatol., 6(11):818-824.

3) Changchien, C. S., Chen, C. L. and Yen, Y. H. (2008). Analysis of 6381 hepatocellular carcinoma patients in southern Taiwan: Prognostic features, treatment outcome, and survival. J. Gastroenterol., 43:159-170. 
4) Hatzakis, A., Van Damme, P., Alcorn, K., Gore, C., Benazzouz, M., Berkane, S. and Buti, M. (2013). The State of Hepatitis B and C in the mediterranean and balkan countries: Report from a summit conference. Journal of Viral Hepatitis, 20(2): 1-20.

5) He, Q. Y., Zhu, R., Lei, T., Ng, M. Y., Luk, J. M., Sham, P., Lau, G. K., and Chiu, J. F. (2008). Toward the proteomic identification of biomarkers for the prediction of $\mathrm{HBV}$ related hepatocellular carcinoma. J. Cell Biochem., 3: 740-752.

6) El-Houseini, M. E., Mohammed, M. S., Elshemey, W. M., Hussein, T. D., Desouky, O. S. and Elsayed, A. A. (2005). Enhanced detection of hepatocellular carcinoma. Cancer Control, 4:248253.

7) Esquela-Kerscher, A. and Slack, F. J. (2006). Oncomirs: microRNAs with a role in cancer. Nat. Rev. Cancer, 6:259-269.

8) Chen, C. J. and Mei-Hsuan, L. (2011). Early diagnosis of hepatocellular carcinoma by multiple microRNAs: validity, efficacy, and costeffectiveness. J. Clin. Oncol., 29(36) 4745-4747.

9) Lawrie, C. H., Gal, S., Dunlop, H. M., Pushkaran, B., Liggins, A. P., Pulford, K., Banham, A. H., Pezzella, F., Boultwood, J., Wainscoat, J. S., Hatton, C. S. and Harris, A. L. (2008). Detection of elevated levels of tumour-associated microRNAs in serum of patients with diffuse large B-cell lymphoma. Br. J. Haematol., 141(5):672-675.

10) Xu, J., Wu, C., Che, X., Wang, L., Yu, D., Zhang, T., Huang, L., Li, H., Tan, W., Wang, C. and Lin, D. (2011). Circulating microRNAs, miR-21, miR122 , and miR-223, in patients with hepatocellular carcinoma or chronic hepatitis. Mol. Carcinog., 50(2): 136-142.

11) Livak, K. J. and Schmittgen, T. D. (2001). Analysis of relative gene expression data using realtime quantitative PCR and the 2-Delta Delta CT method. Methods (San Diego, Calif.), 25(4):402408.

12) Hung, C. H., Chiu, Y. C. and Chen, C. H. (2014). MicroRNAs in hepatocellular carcinoma: carcinogenesis, progression, and therapeutic target. Biomed Res. Int., 2014:486407.

13) Ma, J., Guo, R. and Wang, T. (2015). Let-7b binding site polymorphism in the B-cell lymphomaextra large 3' UTR is associated with fluorouracil resistance of hepatocellular carcinoma. Mol. Med. Rep., 11: 677-681.

14) Giordano, S. and Columbano, A. (2013). MicroRNAs: new tools for diagnosis, prognosis, and therapy in hepatocellular carcinoma. Hepatology, 57: $840-847$.

15) Porta, C., De Amici, M. and Quaglini, S. (2008). Circulating interleukin-6 as a tumor marker for hepatocellular carcinoma. Ann Oncol., 19: 353-358.

16) Mao, Y., Yang, H., Xu, H., Lu, X., Sang, X., Du, S., Zhao, H., Chen, W., Xu, Y., Chi, T., Yang, Z., Cai, J., Li, H., Chen, J., Zhong, S., Mohanti, S. R.,
Lopez-Soler, R., Millis, J. M., Huang, J. and Zhang, H. (2010). Golgi protein 73 (GOLPH2) is a valuable serum marker for hepatocellular carcinoma. Gut, 59(12):1687-1693.

17) Shen, Q., Fan, J. and Yang, X. R. (2012). Serum DKK1 as a protein biomarker for the diagnosis of hepatocellular carcinoma: a large-scale, multicentre study. Lancet Oncol., 13:817-826.

18) Luo, J., Chen, M., Huang, H., Yuan, T., Zhang, M., Zhang, K. and Deng, S. (2013). Circulating microRNA-122a as a diagnostic marker for hepatocellular carcinoma. OncoTargets and Therapy, 6: $577-583$.

19) Giannelli, G., Fransvea, E. and Trerotoli, P. (2007). Clinical validation of combined serological biomarkers for improved hepatocellular carcinoma diagnosis in 961 patients. Clin. Chim. Acta., 383: $147-152$.

20) Giannelli, G., Marinosci, F., Trerotoli, P., Volpe, A., Quaranta, M., Dentico, P. and Antonaci, S. (2005). SCCA antigen combined with alphafetoprotein as serologic markers of HCC. Int. J. Cancer, 117(3):506-509.

21) Ivanovska, I., Ball, A. S., Diaz, R. L., Magnus, J. F., Kibukawa, M. and Schelter, J. M. (2008). MicroRNAs in the miR-106b family regulate p21/CDKN1A and promote cell cycle progression. Mol. Cell Biol., 28: 2167-2174.

22) Petrocca, F., Visone, R., Onelli, M. R., Shah, M. H., Nicoloso, M. S. and de Martino, I. (2008). E2F1-regulated microRNAs impair TGF betadependent cell-cycle arrest and apoptosis in gastric cancer. Cancer Cell, 13:272-286.

23) Yau, W. L., Colin, S., Chi, L., Lui, N., Ariel, K., Man, C., Sylvia, T., Jacky, Y., Jana, Y., Kevin, T., Kwan, M., Ronnie, T. and Roberta, W. (2013). Over-expression of miR-106b promotes cell migration and metastasis in hepatocellular carcinoma by activating epithelial-mesenchymal transition process. PLOS ONE, 8(3): e578828.

24) Zheng, L., Zhang, Y., Liu, Y., Zhou, M., Lu, Y., Yuan, L., Zhang, C., Hong, M., Wang, S. and Li, X. (2015). MiR-106b induces cell radioresistance via the PTEN/PI3K/AKT pathways and p21 in colorectal cancer. J. Transl. Med., 13(1): 252-264.

25) Matsuzaki, J. and Suzuki, H. (2015). Role of microRNAs-221/222 in digestive systems (Review), J. Clin. Med., 4: 1566-1577.

26) Chen, X. M. (2009). microRNA signatures in liver diseases, Wor. J. Gastroenterol., 15(14): 1665-1672.

27) Wong, Q., Ching, A., Chan, A. and Choy, K. W. (2010). MiR-222 overexpression confers cell migratory advantages in hepatocellular carcinoma through enhancing AKT signaling. Clinical Cancer Research, 16(3): 867-876.

28) Qi, J., Wang, J., Katayama, H., Sen, S. and Liu, S. M. (2013). Circulating microRNAs (cmiRNAs) as novel potential biomarkers for hepatocellular carcinoma. Neoplasma, 60: 135-142. 
29) Tan, Y., Ge, G., Pan, T., Wen, D., Chen, L., Yu, X. and Gan, J. (2014). A Serum microRNA panel as potential biomarkers for hepatocellular carcinoma related with hepatitis B virus. PLOS ONE, 9(9): e107986.

30) Li, Y., Weiqi, T., Thomas, W. L. N., Myat, O. A., Shanthi, W., Seng, G. L., and Theresa, M. C. T. (2009). Role of the miR-106b-25 microRNA Cluster in Hepatocellular Carcinoma. Cancer. Science 100 (7): 1234-1242.
31) Shen, G., Jia, H., Tai, Q., Li, Y. and Chen, D. (2013). miR-106b downregulates adenomatous polyposis coli and promotes cell proliferation in human hepatocellular carcinoma. Carcinogenesis, 34(1), 211-219.

32) Connolly, E., Melegari, M., Landgraf, P., Tchaikovskaya, T., Tennant, B. C. and Slagle, B. L. (2008). Elevated expression of the miR-17-92 polycistron and miR-21 inhepadnavirus-associated hepatocellular carcinoma contributes to the malignant phenotype. Am. J. Pathol., 173: 856-864. 